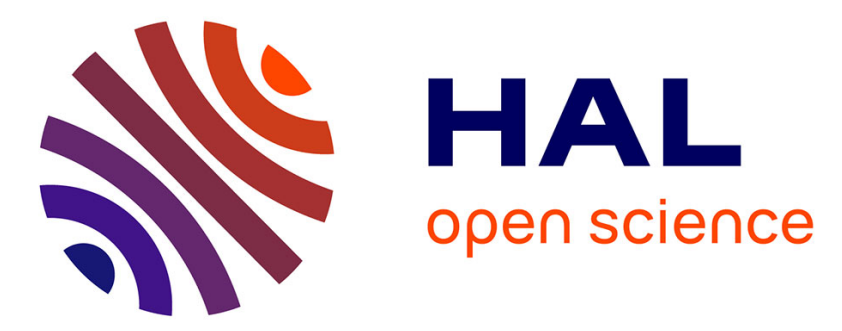

\title{
STARK EFFECT IN QUASI-HYDROGENIC SPECIES
}

E. Luc-Koenig, S. Feneuille, J. Lecomte, S. Liberman, J. Pinard, Ahmed Taleb

\section{To cite this version:}

E. Luc-Koenig, S. Feneuille, J. Lecomte, S. Liberman, J. Pinard, et al.. STARK EFFECT IN QUASI-HYDROGENIC SPECIES. Journal de Physique Colloques, 1982, 43 (C2), pp.C2-153-C2-166. 10.1051/jphyscol:1982212 . jpa-00221823

\section{HAL Id: jpa-00221823 https://hal.science/jpa-00221823}

Submitted on 1 Jan 1982

HAL is a multi-disciplinary open access archive for the deposit and dissemination of scientific research documents, whether they are published or not. The documents may come from teaching and research institutions in France or abroad, or from public or private research centers.
L'archive ouverte pluridisciplinaire HAL, est destinée au dépôt et à la diffusion de documents scientifiques de niveau recherche, publiés ou non, émanant des établissements d'enseignement et de recherche français ou étrangers, des laboratoires publics ou privés. 
JOURNAL DE PHYSIQUE

Colzoque C2, supplément au n¹1, Tome 43, novembre 1982

page $\mathrm{C} 2-153$

\title{
STARK EFFECT IN QUASI-HYDROGENIC SPECIES
}

\author{
E. Luc-Koenig, S. Feneuille, J.M. Lecomte, S. Liberman, J. Pinard and A. Taleb \\ Laboratoire Aimé Cotton, Centre National de Za Recherche Seientifique, \\ Bôtiment 505, 91405 Orsay Cedex, France
}

Résumé - L'analyse des spectres de photoionisation d'atomes à un électron optique en présence d'un champ électrique peut être faite en partant du modèle hydrogénoIde. Le problème de I'effet Stark de l'hydrogène peut être résolu de façon exacte, l'hamiltonien correspondant étant séparable en coordonnées paraboliques. On définit des densités partielles d'états et on montre qu'au-dessus de la limite classique d'ionisation par champ le spectre de l'hydrogène comporte des états quasi-discrets superposés à des états continus, les différents états n'étant pas couplés. Deux expériences concernant les spectres de photoionisation Stark du rubidium montrent l'importance des perturbations liées à l'interaction spin-orbite, comme par exemple la stabilisation d'un état Stark par le champ électrique.

Abstract - Photoionization spectra of one-electron atoms in the presence of an external electric field are analyzed starting from the knowledge of the hydrogen Stark spectrum. The Coulomb-Stark hamiltonian is separable in parabolic coordinates and thus the problem can be exactly solved. Partial densities of continuum states are defined, and it is shown that above the classical field ionization limit the Stark spectrum of hydrogen consists of quasi-stable states embedded in ionization continua. The different states are not coupled to each other. Two experiments on the photoionization Stark spectra of rubidium atoms are reported. These spectra are strongly perturbed by the spin-orbit interaction which is responsible for striking anomalies such as the field induced stabilization of a state.

\section{Introduction.}

Recently a lot of attention has been paid to photoionization spectra of alkaliatoms in the presence of a static electric field $\mathcal{G}$. Various experiments using atomic beams and tunable lasers have been carried out and most of the characteristics of such spectra have been explained, at least qualitatively, starting from the theoretical knowledge of the hydrogen Stark spectrum. However the Stark spectrum of hydrogen strongly differs from those of alkali-atoms. The Coulomb potential possesses a dynamical symmetry which allows states with the same value of $m_{\ell}$ to cross [1]. In alkali-atoms this symmetry is broken and the two concemed states interact and therefore they cannot cross.

The spectrum corresponding to combined Coulombic and Stark potential is a continuum for any value of the energy $E$. However this continuum character does not play a sigmificant role in the subcritical regime, that is for energies smaller than the classical field-ionization threshold $E_{C}=-2 \sqrt{F}$ (atomic units are used and the ionization potential of the unperturbed atom is chosen as the origin for the energy). In the subcritical range the most important features are the shifts and broadenings of the discrete states due to the electric field. The main properties of the Stark spectrum (structure of Rydberg states, anticrossings, oscillator strengths,...) can be obtained very accurately by diagonalizing the energy matrix including only those states which have approximately the same energy, and by taking into account explicitely the non hydrogenic character of the spectrum [2]. 
In the over critical regime $E>E_{C}$, the Stark spectrum of hydrogen consists of quasi-stable states embedded in ionization continua, but, because of the symmetry of the coulomb potential there is no interaction between the different states. Any perturbation, breaking this symmetry, couples states with different field ionization properties and modifies the properties of the spectrum. This paper is essentially devoted to the study of the over critical region where new and rather unexpected phenomena have been observed.

\section{Stark structure and field-ionization properties of hydrogen.}

A - Exact wavefunctions for the hydrogen stark effect.

The Stark hamiltonian of hydrogen is separable in parabolic coordinates and consequently the problem can be exactly solved [3], inasmuch as the relativistic corrections are neglected.

The hamiltonian for a hydrogen-like atom of nuclear charge $Z$ in an external electric field 5 directed along the tz-axis is

$$
\mathrm{H}_{0}=-\frac{1}{2} \nabla^{2}-z / r+\xi_{z} \quad \text {. }
$$

It commutes with both z-projections of the kinetic moment operator $\vec{I}$ and of the generalized Runge-Lenz vector [1]

$$
\vec{A}=(\vec{p} \times \vec{I}-\vec{L} \times \vec{p}) / 2-\overrightarrow{z r} / r-(\vec{r} \times \vec{F}) \times \vec{x} / 2 \text {. }
$$

The mutually commuting operators $\mathrm{H}_{0}, \mathrm{~L}_{z}$ and $\mathrm{A}_{\mathrm{z}}$ form a complete set of operators [5], the eigenvalues of which $\left(\mathrm{E}, \mathrm{m}_{\ell}\right.$ and $\left.\left(2 z_{1}-\mathrm{z}\right)\right)$ can be used to determine orthogonal eigenstates of $H_{O}$. By using the parabolic coordinates [3] $\xi=r+z$, $\eta=r-z, \Phi=\tan ^{-1} \mathrm{y} / \mathrm{x}$, the wavefunction is written as

$$
\Psi(\xi, \eta, \Phi)=(\xi, \eta)^{-1 / 2} F(\xi) G(\eta) e^{i m_{\ell} \Phi}(2 \pi)^{-1 / 2}
$$

and the resulting one-dimensional equations for $\xi$ and $\eta$ are

$$
\begin{aligned}
& \frac{d^{2}}{d \xi^{2}} F(\xi)+\left(-\frac{m_{l^{2}}^{2}}{4 \xi^{2}}+\frac{z_{1}}{\xi}+\frac{E}{2}-\frac{\mathscr{F}}{4} \xi\right) F(\xi)=\left(\frac{d^{2}}{d \xi^{2}}+2 T_{\xi}\right) F(\xi)=0 \\
& \frac{d^{2}}{d \eta^{2}} G(\eta)+\left(-\frac{m_{l^{-1}}^{2}}{4 \eta^{2}}+\frac{z_{2}}{\eta}+\frac{E}{2}+\frac{G}{4} \eta\right) G(\eta)=\left(\frac{d^{2}}{d \eta^{2}}+2 T_{\eta}\right) G(\eta)=0
\end{aligned}
$$

The separation constants $z_{1}$ and $z_{2}$ represent the "effective charges" respectively associated to $\xi$ and $\eta$ motions; they are coupled through the relation $z_{1}+z_{2}=z$.

Solutions of these equations can be obtained either numerically [5] or semianalytically from a modified WKB treatment [6]. These methods do not involve expansions in powers of $\mathscr{F}$ and are valid for any values of $\mathrm{E}$ and $F$.

The effective kinetic energies $T_{\xi}$ and $T_{n}$ (Eq. 4) differ in the sign of the electric potential and this determines the difference in the behaviour of $F(\xi)$ and $G(\eta)$. Indeed classically the electron is located in the regions of space where $T_{\xi}$ and $T_{\eta}$ are simultaneously positive. Owing to the boundary condition for the variable $\xi, z_{1}$ is the discrete eigenvalue of the bound-state problem. For fixed values of $\mathrm{E}, \mathscr{F}$ and $\left|\mathrm{m}_{\ell}\right|$ the different eigenvalues $z_{1}\left(\mathscr{G}_{\mathrm{F}}, \mathrm{E} ; \mathrm{n}_{1},\left|\mathrm{~m}_{\ell}\right|\right)$ can be characterized by the integer $n_{1}$ which is equal to the number of nodes of the function $F(\xi)$ associated with the bound motion. The motion in $n$ is a free motion, consequently all continuum properties of the wavefunction $\Psi$ are included in the $G(\eta)$ function. Iet us remark that the motion in $\eta$ is entirely determined by the values of the quantum numbers $\mathrm{E}, \mathrm{m}_{\ell}$ and $\mathrm{n}_{1}$, or by the equivalent set $\mathrm{E}$, $\mathrm{m}_{\ell}, \mathrm{z}_{1}$. Indeed once $z_{1}$ is found, the value of $z_{2}$ is obtained from (Eq. 5). For any field stength $\mathscr{F}$, the quantum numbers $m_{\ell}$ and $Z_{q}$ takes only discrete values, but the schrödinger equation has a continuous spectrum with respect to the energy $\mathbf{E}$.

B - Parabolic ionization thresholds at negative energy. From the study of $T_{\eta}$ it is possible to define parabolic ionization thres- 
holds which depend not only on the field strength $F$ or on the total energy $E$, but also on $\left|m_{\ell}\right|$ and on the separation constant $z_{2}$ (i.e. on $n_{1}$ ). Typical drawings of $T_{\eta}$ are presented in Figure 1. For a fixed value of $\left|\mathrm{m}_{l}\right| \quad\left(\left|\mathrm{m}_{l}\right|>1\right)$ and not too high a value of $n_{1}$, two very different types of drawings can be obtained. For an energy smaller than $g \frac{p}{c}\left(F_{1} ; n_{1}, m_{l}\right), T_{n}$ is positive in two distinct ranges for $\eta$ : the first one corresponds to small $\eta$ values, the second one to large $\eta$ values. These two regions are separated by a potential barrier where $T_{\eta}$ is negative. For such states spontaneous ionization is possible in a quantum mechanical treatment owing to tunnelling through the barrier. As the energy increases the barrier becomes narrower and lower until it finally disappears at $E=E_{c}^{P}\left(G_{F} ; n_{1},\left|m_{\ell}\right|\right)$. For greater energy values there is no potential barrier and ionization is possible even according to classical mechanics. The quantity $\mathrm{E}_{\mathrm{c}}^{\mathrm{p}}\left(\mathscr{F}_{7} ; \mathrm{n}_{1},\left|\mathrm{~m}_{\ell}\right|\right)$ represents the parabolic ionization threshold for states $\mathrm{n}_{1},\left|\mathrm{~m}_{\ell}\right|$. For states $\left|\mathrm{m}_{\ell}\right|=1$ the parabolic ionization threshold is given by [3]

$$
4 F_{2}=\left[\mathrm{E}_{\mathrm{C}}^{\mathrm{P}}\left(G_{;} ; \mathrm{n}_{1},\left|\mathrm{~m}_{\ell}\right|=1\right)\right]^{2}
$$

where $z_{2}$ depends implicitly on $\mathscr{F}$. For the different states of a highly excited manifold with $n \gg\left|m_{\ell}\right|$, it can be shown by using the result of the perturbation theory that for the lowest state $n_{1}=0 \quad z_{2}$ does not differ to much from 1 : for such a state the parabolic ionization threshold is approximately equal to the classical ionization energy $E_{c}=-2 \sqrt{g^{4}}$. On the contrary for the most excited state $n_{1} \sim n$ and $n_{2}=0, z_{2}$ is very small and of the order of $1 / n$. Then the parabolic ionization threshold is given by [7]: $\mathbb{F} \sim \mathrm{E}_{\mathrm{c}}^{\mathrm{p}} \mathrm{n} / 4 \sim 0.21\left(\mathrm{E}_{\mathrm{c}}^{\mathrm{p}}\right)^{8 / 5}$. For fixed $\mathscr{F}$ and $m_{\ell}$, the parabolic ionization threshold increases with $n_{1}$ and becomes greater than the zero-field ionization threshold for sufficiently high values of $n_{1}$.
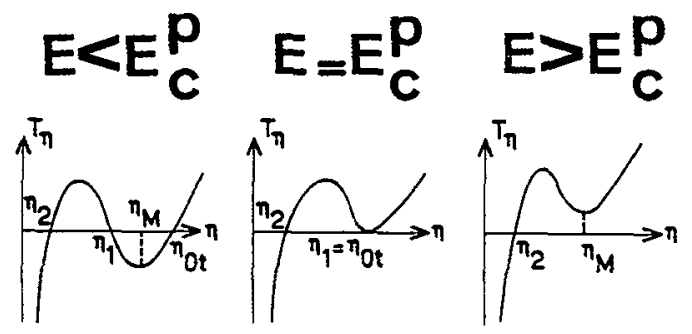

Figure 1 - Typical drawing of the effective kinetic energy for states $\left|\mathrm{m}_{\ell}\right| \geqslant 2 . \mathrm{E}_{\mathrm{C}}^{\mathrm{p}}$ is the parabolic critical energy.

\section{C - Density of states.}

The normalization of the wavefunction $\Psi\left(\mathscr{F}, \mathrm{E} ; \mathrm{n}_{1}, \mathrm{~m}_{\ell}\right)$ per unit energy range is determined by an asymptotic condition on $\eta$, which corresponds to the value $1 /(2 \pi)$ for the outgoing flux I out through a surface of constant $\eta(\eta \rightarrow+\infty)$. $[4,6]$. Near the origin, the amplitude of $\Psi$ is given by :

$$
\Psi\left(\Psi, E ; n_{1}, m_{\ell}\right)=\left[\mathcal{E}\left(\Psi, E ; n_{1},\left|m_{\ell}\right|\right]^{1 / 2}\left(\xi_{n}\right)^{\left|m_{\ell}\right| / 2} e^{i m} \ell^{\Phi}(2 \pi)^{-1 / 2}\right. \text {. }
$$

For chosen values of $F,\left|m_{l}\right|$ and $n_{1}, \mathscr{C}$ is a continuous function of the energy, which is called the partial density of states with quantum numbers |ml| and $n_{1}$. This function does not vary monotonically as it is shown in Figure 2 . For energies smaller than the parabolic critical energy $E g\left(F_{;} n_{1},\left|m_{\ell}\right|\right)$ and for a small nq-value, $\ell$ behaves as a quasi discrete spectrum : it is vanishingly small except in very narrow energy ranges $E_{n}$ where it presents very sharp peaks with a Iorentzian profile of width $\Gamma_{n}$. The ${ }^{2}$ different resonances occurring in the curve $\ell\left(\mathscr{F}, E ; n_{1},\left|m_{\ell}\right|\right)$ can be labelied with the quantum number $n_{2}$ which is equal to the number of nodes of the $G(\eta)$ function inside the classical bound region $\left(\eta_{2}<\eta<\eta_{1}\right.$ Figure 1). The energies $E_{n_{2}}$ of the quasi discrete states can be determined very accurately from perturbation theory [3]. The width of the resonance, which is related to the probability of ionization of the quasi-stable state by tunnelling through the potential barrier, increases with the energy but remains 
smaller than $10^{-2} \mathrm{~cm}^{-1}$. For energies greater than $E_{\mathrm{c}}^{\mathrm{p}}\left(\mathscr{G} ; \mathrm{n}_{1},\left|\mathrm{~m}_{\ell}\right|\right)$, the partial density of states looks really like a continuous spectrum since no potential barrier remains in the $n$-motion. A small number ( 1 or 2 ) of broad unresolved structures can be observed above $E_{c}^{p}$, but these structures disappear rapidly with increasing energy. The partial density of continuum states becomes very small at the energy $\mathrm{E}_{\mathrm{d}}\left(G ; \mathrm{n}_{1},\left|\mathrm{~m}_{\ell}\right|\right)$ which is greater than the zero field ionization energy. The disappearance of the states $n_{1},\left|m_{\ell}\right|$, for $E>E_{d}$ can be explained by recalling that $\ell$ is directly related to the probability of finding the electron near the nucleus (Eq. 7); consequently the partial density of states $n_{1},\left|m_{\ell}\right|$ is not negligible only at such energies that $T_{\xi}$ and $T_{\eta}$ are simultaneously positive for small $\xi$ and $\eta$. These conditions are fulfilled for $0 \leqslant z_{1} \leqslant z$ because the "effective charges" associated with the $\xi$ and $\eta$ motions correspond then to attractive

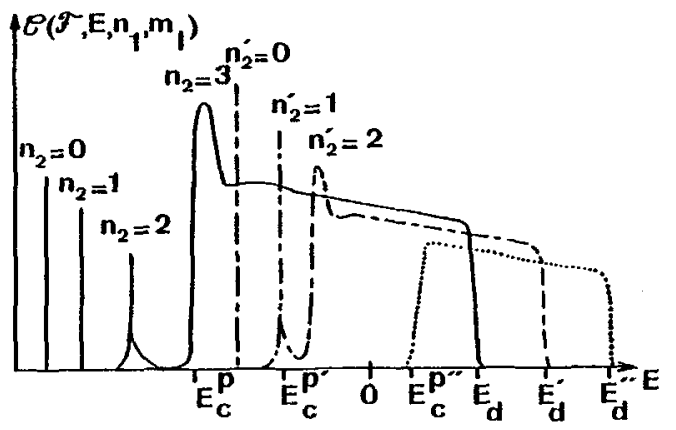

Figure 2 - Typical energy-dependence of the partial density of states for different $n_{1}$ - values and for a given field strength.

potentials. For fixed $n_{1}$ and $\left|m_{l}\right|, z_{1}$ is a decreasing function of $E$, which vanishes for $\mathrm{E}=\mathrm{E}_{\mathrm{d}}\left(\mathbb{F} ; \mathrm{n}_{1},\left|\mathrm{~m}_{\ell}\right|\right)$. In a similar way, one can explain that a state with a sufficiently high value of $n_{1}$ appears at a positive energy $\mathbb{E}_{c}^{p}\left(\mathscr{F}_{;} ; n_{1},\left|m_{\ell}\right|\right)$ corresponding to $z_{1}=Z$. For such a high $n_{1}$-value, $\mathscr{C}$ exhibits a very broad structure extending from $\mathrm{E}_{\mathrm{C}}^{\mathrm{p}}$ to $\mathrm{E}_{d}$, but no resonance exists at $E<\mathrm{E}_{\mathrm{c}}^{\mathrm{p}}$. (Fig. 2). Let us remark that the energies $E_{d}\left(F ; n_{1},\left|m_{l}\right|\right)$ and $E_{c} p\left(F ; n_{1},\left|m_{l}\right|\right)>0$, which correspond to well-defined values for $z_{1}$ (respectively 0 and $z$ ), can be obtained by considering the equation $(4 a)$ as a one-dimensional eigenvalue problem with respect to the energy $E$.

The total density of states with a given $m_{\ell}$ value, $\ell\left(\mathscr{F}, E ; m_{\ell}\right)$ is the sum over all partial densities $\ell\left(\mathscr{F}_{\ell}, \mathrm{E} ; \mathrm{n}_{1}, \mathrm{~m}_{\ell}\right)$. Above the zero-field ionization limit, the total densities of states consist of very broad and numerous structures. Consequently the energy dependence of the total density of states exhibits oscillations with an almost negligible modulation depth even at relatively high field strength $(\sim 100 \mathrm{kV} / \mathrm{cm})$. Furthermore no significant difference is observed in $\ell\left(\mathbb{F}, E ;\left|\mathrm{m}_{\ell}\right|\right)$ as $\left|\mathrm{m}_{\ell}\right|$ varies.

D - Density of oscillator strengths. Recent experiments on the Stark effect of one-electron spectra, such as $\mathrm{Rb}[8]$ and Na $[9,10]$ have shown that the photoionization cross section presents oscillations extending near and above the zero-field ionization limit, these structures depending strongly on the polarization of the exciting light. These experimental data are related to the density of oscillator strengths for single-photon excitation from a weakly excited state of energy $E_{i}$, considered as a discrete state $\varphi_{i}$ even in the presence of an electric field. The partial density of oscillator strengths for a transition from $\varphi_{i}$ towards the continum state $\Psi\left(\mathscr{F}, \mathrm{E} ; \mathrm{n}_{1}, \mathrm{~m}_{\ell}\right)$ is defined by

$$
\mathrm{df} / \mathrm{dE}\left(\mathscr{F}, \mathrm{E} ; \mathrm{n}_{1}, \mathrm{~m}_{\ell}\right)=2 / 3\left(\mathbb{E}-\mathrm{E}_{i}\right)\left|\left\langle\varphi_{i}\left|\mathrm{r}_{\mathrm{q}}\right| \Psi\left(F, \mathrm{E} ; \mathrm{n}_{1}, \mathrm{~m}_{\ell}\right)\right\rangle\right|^{2}
$$

where $r_{q}=\mathbf{z}$ for $\pi$-polarization and $(x \pm i y) / \sqrt{2}$ for $\sigma^{ \pm}$polarizations. Experimentally, starting from a pure well defined initial state $\varphi_{i}$ and using polarized light, one can observe only the total density of oscillator strengths 
$\mathrm{df} / \mathrm{dE}\left(\bar{F}, E ; \mathrm{m}_{\ell}\right)$ which is the sum over the partial densities of oscillator strengths for all $n_{1}$-channels.

The partial density of oscillator strengths does not depend directly on the partial density of states ; it is a nondiagonal quantity in which two different orbitals $\varphi_{i}$ and $\Psi$ appear. Thus the energy-dependence of $\zeta$ and $\mathrm{df} / \mathrm{dE}$ can be very different. Therefore for interpreting the photoionization Stark spectra near the ionization limit, the important quantity to be considered is the oscillatorstrength density rather than simply the density of states. This important result was stressed from earlier numerical calculations on the photoionization spectra of the ground state of hydrogen in the presence of an external electric field [11] : only the explicit calculation of the energy dependence of the total density of oscillator strengths can give reliable informations on the structure of the photoionization spectra near and above the zero-field ionization limit. Nevertheless some qualitative predictions can be obtained from very simple arguments.

Firstly, important cancellation effects occur in the caloulation of the transition matrix element $\left\langle\varphi_{i}\left|r_{q}\right| \Psi\left(\mathscr{F}^{\pi}, E ; n_{1}, m_{\ell}\right)\right\rangle$ when there exist well defined symmetry properties. The wavefunction $\Psi\left(F, E ; n_{1}, m_{\ell}\right)$ has general ly no symmetry property except in the energy range $\mathrm{E}_{\sim} \sim \mathrm{E}_{\mathbb{S}}\left(\mathscr{F} ; \mathrm{n}_{1}, \mathrm{~m}_{2}\right)$ in which $z_{1} \sim \mathrm{z}_{2} \sim \mathrm{z} / 2$, where $\Psi$ is symmetrical with respect to the $z=0$ plane. Indeed, in the neighbourhood of the nucleus, $T_{\mathcal{F}}$ and $\mathbb{T}_{\eta}$ are equal, inasmuch as the Stark potentials can be neglected in Eq. 4. The enexgy $\mathrm{E}_{\mathrm{S}}\left(\mathscr{F}_{F} ; n_{1}, \mathrm{~m}_{\ell}\right)$ is located midway between $\mathrm{E}_{\mathrm{C}}^{\mathrm{p}}\left(\mathscr{F}_{F} ; \mathrm{n}_{1}, \mathrm{~m}_{\ell}\right)$ and $\mathbb{E}_{\mathrm{d}}\left(\mathscr{F} ; \mathrm{n}_{1}, \mathrm{~m}_{\ell}\right)$. The wavefunction $\varphi_{i}$ for the weakly excited state $\ell_{j} m_{l_{i}}$, is symmetrical or antisymmetrical with respect to the $z=0$ plane according ${ }^{2}$ to whether $\ell_{i}+m \ell_{j}$ is even or odd. The transition operator is antisymmetrical for a $\pi$-polarization $\left(\mathrm{m}_{\ell_{j}}=\mathrm{m}_{\ell}\right)$ or symmetrical for a $\sigma$-polarization $\left(\left|\mathrm{m}_{\ell}-\mathrm{m}_{\ell_{j}}\right|=1\right)$. It follows that the paitial density of oscillator strengths vanishes at the $i_{\text {energy }}$ $\mathrm{E}_{\mathbf{S}}$ if one considers absorption either from a symmetrical state with $\pi$-polarized

Figure 3 - Energy-dependences of the partial density of excited states (--) and the partial density of oscillator strengths (_. in the photoabsorption spectrum from the ground state of hydrogen towards excited states with a given $n_{1}$-value. The total density is also presented. $0 \rightarrow 0$ and $0 \rightarrow 1$ correspond respectively to $\pi$ and $\sigma$ polarizations. $\mathscr{G}=77 \mathrm{~kW} / \mathrm{cm}$.

light, or from an antisymmetrical state with $\sigma$-polarized light. In the other two cases no cancellation effects appear in the calculation of the transition matrix element at $E=E_{\mathbb{S}}$. Figure 3 presents the energy dependences of both the partial density of continuum states and the partial density of oscillator strengths in the photoabsorption spectrum from the symmetrical ground state $\ell_{i}=m_{\ell_{i}}=0$ of hydrogen for $\bar{F}=77 \mathrm{kV} / \mathrm{cm}$, and with a $\pi$ - and a $\sigma$-polarization of the İght. For the $\pi$ polarization, $d f / d E$ vanishes midway between $\mathrm{E}_{\mathrm{C}}^{\mathrm{p}}$ and $\mathrm{E}_{\mathrm{d}}$, and therefore exhibits a relatively narrow resonance at $\mathrm{E}_{\mathrm{C}}^{\mathrm{p}}$. On the contrary for the a-polarization no cancellation effects occur and $\mathscr{C}$ and $\mathrm{df} / \mathrm{dE}$ are almost proportional. ConsequentIy the total density of oscillator strengths presents a significantly modulated structure in the $\pi$-spectrum in contrast to the almost structureless o-spectrum. In the photoionization of an excited state $\ell_{j}, \mathrm{~m}_{\ell_{i}}$ one generally reaches two different values of $l, l=\ell_{i} \pm 1$, in the final state. "Then interference effects between the two channels $\ell_{i}+1$ and $\ell_{i}-1$ appear, and they are associated with additional cancellation effects in the partial density of oscillator strengths $\mathrm{df} / \mathrm{dE}\left(\bar{F}, E ; n_{1}, m_{\ell}\right)$, for example at $E=E_{a}$ such as $Z_{1}=Z_{1}^{a}$. By studying the 
effective kinetic energies ( $\mathrm{Eq} .4$ ), it can be show that $\mathrm{df} / \mathrm{dE}$ has a second minimum at $E=E_{b}$ corresponding to $Z_{1}=Z-Z_{1}^{a}$. When $d f / d E$ has several minima separated by more or less sharp peaks, then the total density of oscillator strengths exhibits oscillations with a significant modulation depth. Destructive interference effects between the two channels $\ell_{i}+1$ and $\ell_{i-1}$ appear in the spectra obtained with $\pi$ - or $\sigma$-polarized light, if the magnetic quantum number of the lower state verifies respectively $\left|\mathrm{m}_{\ell_{i}}\right| \leqslant \ell_{i}-1$ or $\left|\mathrm{m}_{\ell_{i}}\right| \leqslant \ell_{i}-2$. The presence of cancellam tions in the partial density of states, which do not arise from symmetry properties, has been pointed out in the exact calculation of the two step photoionization spectra from the $3 \mathrm{p} \mathrm{m} \mathrm{g}_{\mathrm{i}}$ state of hydrogen in the presence of an electric field [12]. The results are reported in Figure 4 for different polarizations of the exciting and photoionizing light. For the $\pi \pi$ and the $\sigma^{+} \sigma^{-}$spectra, i.e. for the transitions $3 \mathrm{p} \mathrm{m}_{\ell_{i}}=0 \rightarrow \mathrm{m}_{\ell}=0$ and $3 \mathrm{p} \mathrm{m} \ell_{i}=1 \rightarrow \mathrm{m}_{\ell}=0$, the partial cross-sections have two minima, because the continua ${ }^{i} s$ and $d$ are reached. On the contrary for the $\sigma^{+} \sigma^{+}$ spectrum (transition $3 p \mathrm{~m}_{\ell}=1 \rightarrow \mathrm{m}_{\ell}=2$ ), $\mathrm{d} f / \mathrm{dE}$ does not vanish since only the $\mathrm{d}$ continum is populated and there is no cancellation effect due to symmetry properties at $E=E_{\mathrm{S}}$. For the spectrum $\sigma^{+} \pi\left(3 p \mathrm{~m}_{l_{j}}=1 \rightarrow \mathrm{m}_{\ell}=1\right)$, df $/ \mathrm{dE}$ has a single minimum at $E=E_{\mathfrak{S}}$ due to symmetry properties. " ${ }_{\text {For }}$ the first two cases, important modulations are observed in the total density of oscillator strengths, while in the third one the total spectrum is very smooth and in the last one only a slightly modulated structure is observed.

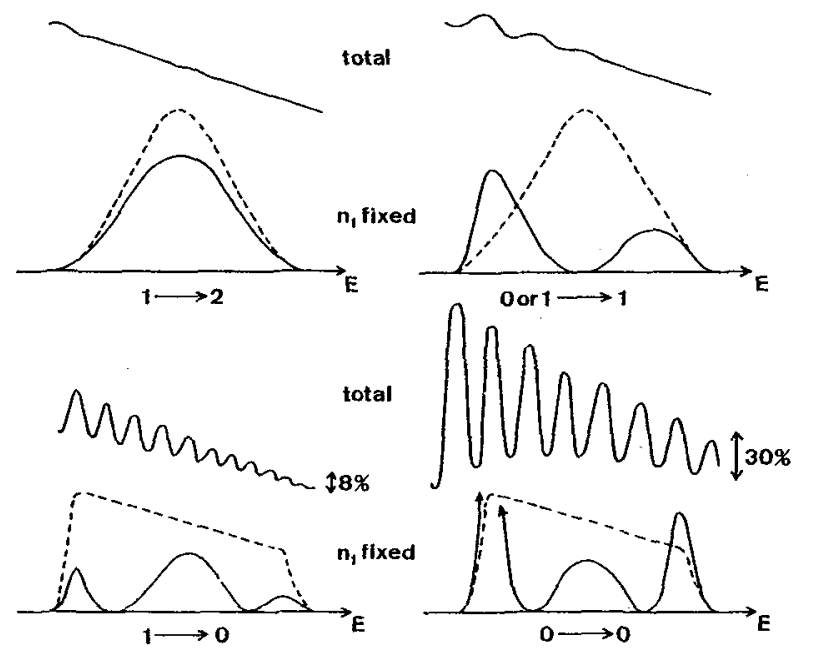

Figure 4-The same as in figure 3 but for photoabsorption from the $3 p$ state of hydrogen. $m_{\ell_{j}} \rightarrow \mathrm{m}_{\ell}$ indicates the magnetic orbital quantum numbers of the $3 p$ and of the excited-state.

Each modulation above the zero-field ionization limit corresponds to the appearance of a new channel associated with a higher n1-value. Consequently each peak is located at the parabolic ionization threshold of the corresponding $n_{1}-c h a n n e l$. As said previously the position of the structures can be obtained by determining the eigenvalues of the one-dimensional potential given for $\left|m_{\ell}\right|=1$ by $(-Z / z+\$ z)$. This model potential was introduced from semi-classical arguments [8]; the exact calculation allows us to justify the results obtained in the classical approach. The structures in the total density of oscillator strengths are very sharp when continuum states $\mathrm{m}_{\ell}=0$ are excited, because the partial density of states $\mathrm{m}_{\ell}=0$ rises very abruptly towards its maximum value at $E=E_{C}^{p}$ (Figures 3 and 4 show the difference in behaviour of the partial density of states with $m_{l}=0$ and $m_{l} \neq 0$ ). At $E=E_{C}^{p}$ the wavefunction of a state $m_{\ell}=0$ is highly concentrated along the positive z-axis with $n_{1}$-nodes, and it corresponds to the classical stable trajectory which undergoes cyclical motion about the positive z-axis [8]. These considerations allow us to explain why structures in the stark photoionization spectra are preferentially 
observed whenever upper states, $m_{l}=0$ are excited, specially for $m_{l}=0 \rightarrow m_{l}=0$ transitions (see Figures 3 and 4). In such cases, all the three functions occurring in the transition matrix element $\left(\varphi_{i}, \Psi, z\right)$ take very large values on the z-axis, and the partial density of oscillator strengths has very high maxima, specially at $\mathrm{E}=\mathrm{E}_{\mathrm{C}}^{\mathrm{P}}$. In the other cases, these three functions have significant values in different spatial ranges so $\mathrm{df} / \mathrm{dE}$ undergoes considerable spatial smoothing of the interference effects.

\section{Non-hydrogenic effects in the Stark photoionization spectra of Rb.}

When a perturbation breaks the dynamical symmetry specific to the Coulomb-Stark potential a different field ionization process occurs [13] : states which are stable with respect to pure tunnelling effect, can ionize due to mixing with an already ionizing state. This process appears in many-electron atoms in which the central potential acting on the outer electron is no longer coulombic. Thus states with the same $m_{l}$ value but with different $n_{1}$ quantum number, can be strongly mixed. The symmetry can also be broken by relativistic interactions coupling states with the same $m_{j}$ value. This kind of mixing exists in all atoms even in hydrogen. Any perturbation which couples a stable state to a continuum, can strongly perturb the photoionization spectra. In this section we describe two recent experiments dealing with the Stark photoionization spectra of $R b$. Rather unexpected phenomena have been observed, which have been attributed to symmetry breaking due to very weak perturbation induced by the spin-orbit interaction.

A - Field-induced stabilization of a well defined stark state of $\mathrm{Rb}$.

In this experiment [14], Rb atoms in their ground state are photoionized by the light of a single-mode pulsed dye laser propagating in a direction perpendicular to both the atomic beam and the electric field. The produced ions are counted and numerous high-resolution photoionization spectra are recorded for different field strengths $\mathscr{F}$. The experimental map of excited states observed with a $\pi$-polarization of the light is presented in Fig. 5. The studied region lies very close to the saddle-point line $(E=-2 \sqrt{F})$. Studying the time of arrival of the produced ions onto the detector after the short light pulse excitation, an anomalous dispersion in time is observed. This anomaly is located very precisely on curve A.

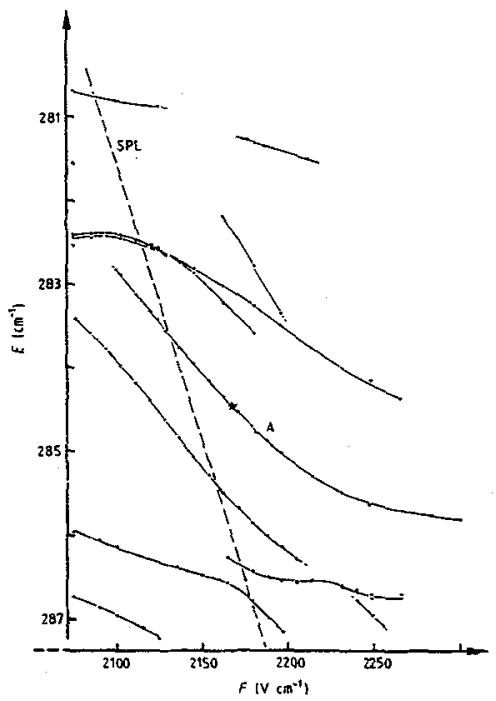

Figure 5 - Experimental map of the states of rubidium in a static electric field observed from the ground state with a $\pi$-polarization.

The lifetime of state A exhibits an anomalous behaviour; the location is indicated by the star.

SPI Saddle point line

$$
E=-2 \sqrt{g^{4}}
$$




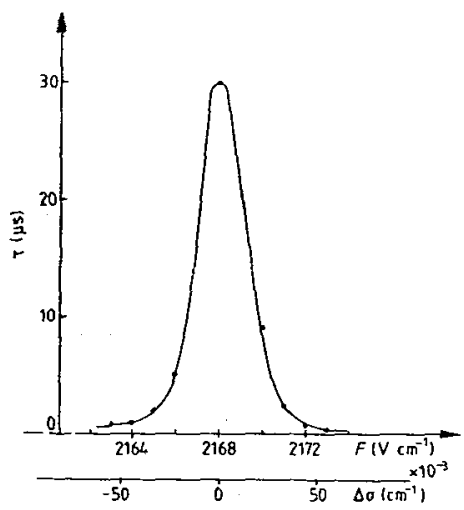

\section{Figure 6}

Lifetime of the state A against the electric field $\mathbb{Y}$.

The measurement of the lifetime of the state $A$ as a function of the electric field exhibits an important increase (a factor of 30) in a very narrow range of field strength $(\Delta \mathbb{F} \sim 8 \mathrm{~V} / \mathrm{cm})$ as it is shown in Fig. 6. The field-ionization process of the state A cannot be ascribed to tunnelling effect, because the ionization rate does not increase regularly with increasing field strength. It arises then necessarily from state mixing. We have searched for a neighbouring stable state by recording the spectra obtained by adding a strong pulsed electric field, but no other stable state has been observed. So the anomaly must be explained as resulting from the mixing of the state A with an unstable state. In fact the mixing of a stable state with a rapidly ionizing state may sometimes lead to a localized decrease in the field ionization rate of the stable state, as it is shown by studying a rather simple model. We consider two non-interacting Stark states of hydrogen, which cross at a given field strength $\mathscr{F}_{C}$; the difference in the energies of these states varies linearly with $\mathscr{g}$ :

$$
\mathrm{E}_{1}(\mathscr{F})-\mathrm{E}_{2}(\mathscr{F})=\mathrm{A}\left(\mathscr{F}-\mathscr{F}_{\mathrm{c}}\right) \text {. }
$$

The ionization rate for the two states is determined by tunnelling and we suppose that the first state is slightly unstable : its width $\Gamma_{1}$ increases rapidly with S5 $[15]:$

$$
1 / 2 \Gamma_{1}(\Psi)=\alpha \exp (\beta \Phi) \text {. }
$$

This resonance is associated with a narrow continuum with a density of states [5] given by a normalized Iorentzian function

$$
\zeta_{1}(E, G)=\frac{1}{2} \Gamma_{q}(\mathscr{G}) /\left[\left(\Phi-E_{1}(\mathscr{F})\right)^{2}+\left(\Gamma_{1}(\xi) / 2\right)^{2}\right] \text {. }
$$

As $F^{\prime}$ increases the width of the Iorentzian increases and its height decreases. In the neighbourhood of the nucleus the wavefunction of this state, normalized per unit energy, is given by (cf. Eq. 7) :

$$
\Psi_{1}(\mathrm{E}, \vec{\Psi}, \vec{r})=\left[b_{1}(E, \mathscr{G})\right]^{1 / 2} \varphi_{1}(\vec{r}) \quad, \quad|\vec{r}| \sim 0 .
$$

We suppose the second state to be very stable : it would correspond to a quasi-discrete state with a negligible width. This second state is described by the wave.. function $\varphi_{2}(\vec{r})$ which does not depend on $G_{t}$.

In the real atom these two states are coupled through a perturbation $W(\vec{r})$ which vanishes for large $|\vec{r}|$. Due to this coupling the quasi-discrete state ionizes and its width is equal to [16]:

$$
\Gamma_{2}(\vec{G}) \propto\left|\left\langle\Psi_{1}(\mathrm{E}, \vec{G}, \overrightarrow{\vec{r}})|W(\vec{r})| \varphi_{2}(\vec{r})\right\rangle\right|_{\mathrm{E}_{\mathrm{I}}=\mathrm{E}_{2}}^{2}(\vec{F})
$$


At a given field $F$, the width of the stable state is proportional to the density of states describing the broad state measured at the energy of the discrete state. It is obvious that $\Gamma_{2}(\mathscr{F})$ does not increase regularly with increasing $\mathscr{G}$. Cenerally the lifetime $\tau_{2}(\mathscr{F}) \propto\left[\Gamma_{2}(F)\right]^{-1}$ decreases in a given range of $\mathscr{F}$, a phenomenon which can be explained simply by diagonalizing the complex-energy matrix formed with, the unperturbed energies of the two states, their decay rates and their mutual coupling [17]. For particular values for the set of parameters A, $F_{c}, \alpha$ and $\beta$ a more irregular variation is observed as it is shown in Figure 7 : in addition to the previously described minimum, there is a narrow maximum followed by a second minimum. In a given range of field strengths, $\tau_{2}$ presents a maximum corresponaing to a stabilization of the state. In conclusion, by taking into account explicitely both the field and energy dependences of the density of states associated with the unstable state it is possible to show that the anticrossing of this state with a stable state leads sometimes to a very well located increase of the lifetime of the stable state. To interpret the anomaly in the field-ionization

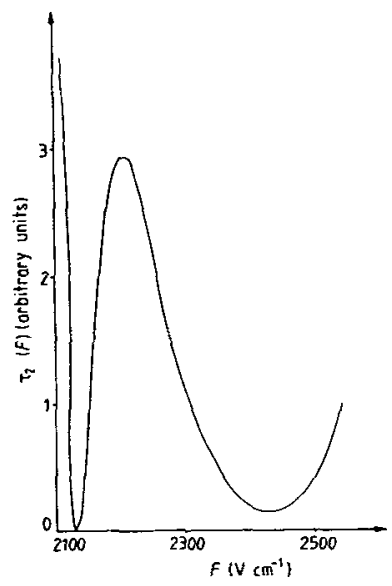

Figure 7 - Calculated field dependence for the lifetime $\tau_{2}$ of the stable state $(21,4,1)$, coupled to the unstable state $(23,0,0)$ through the spin-orbite interaction.

$$
\begin{array}{ll}
A=-0.0241 \mathrm{~V}^{-1} & \mathscr{F}=2132 \mathrm{~V} / \mathrm{cm}^{-1} \\
\alpha=9.13410^{-30} \mathrm{~cm}^{-1} & \beta=0.0238 \mathrm{~V}^{-1} \mathrm{~cm}
\end{array}
$$

rate of the state $A$, as due to the process described above, it is necessary to identify, in the corresponding range $\left(\mathrm{E}, F^{\prime}\right)$ of the hydrogen spectrum, two states with different field-ionization rates. If the spin-orbit interaction is negligible only $\mathrm{m}_{\ell}=0$ states can be excited from the ground state $\ell_{i}=\mathrm{m}_{\ell_{i}}=0$ by using a $\pi-$

Fisure 8 - Stark structure of $m_{\ell}=0$ states of hydrogen.

The states are labelled by $\left(n, n_{1}, m_{\ell}\right)$

-...- Experimentally observed state A

$(21,4,1)$ state of hydrogen

mumb Anomalous behaviour in the lifetime of the state A.

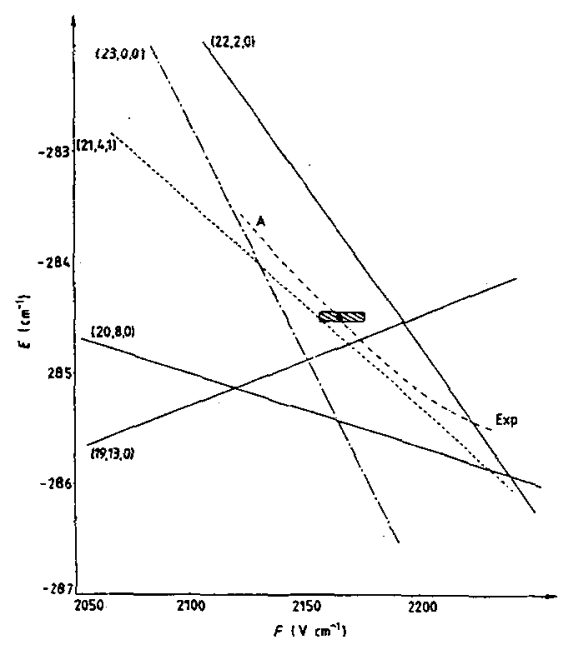


polarization of the exciting light. We have identified all $\mathrm{m}_{\ell}=0$ states of hydrogen present in the range of energy and field strength studied. They are very stable states $(\tau>10-3 \mathrm{~s})$ except for the state $n=23, n_{1}=0$ which is relatively unstable : its lifetime decreases from $3 \cdot 10^{-8}$ to $2.10^{-9} \mathrm{~s}$ when $F$ increases from 2050 to $2250 \mathrm{~V} / \mathrm{cm}$. This state, associated with a very low density of states, cannot be efficiently populated by absorption from the ground state, and is not observed in the experiment. There is no $m_{l}=0$ state corresponding to the observed state $A$, but for relatively heavy atoms such as $\mathrm{Rb}$ the spin-orbit interaction cannot be neglected and $m_{j}$ rather than $m_{\ell}$ is an exact quantum number. Therefore with $\pi-p \circ l a r i-$ zed light it is also possible to excite states $m_{j}=1 / 2 \quad m_{\ell}=1$ from the ground state. We have shown that the location of the very stable state. $n=21, n_{1}=4$ m $m_{l}=1$ does not differ significantly from the recorded position of the stable state A (Fig.8). Furthermore assuming that the anomaly observed in the lifetime of the state A arises from the level anticrossing effect due to the spin-orbit interaction involving the stable state $(21,4,1)$ and the slightly unstable state $(23,0,0)$, we have determined the values of the parameters $\alpha, \beta, \mathscr{F}_{C}$ and $A$ occurring in the previously described model and we have verified that this set of parameters is associated with an important increase of the lifetime of the stable state in a very narrow range of field strengths. Such a result supports the validity of the theoretical interpretation of the anomalous behaviour in the field-ionization properties of the state A.

$$
\begin{aligned}
& \mathrm{B}-\text { Photoionization Stark spectra from the excited state } 5{ }^{2} \mathrm{P}_{3 / 2} \quad \mathrm{~F}=\mathrm{M}_{\mathrm{F}}=4 \\
& \text { of }{ }^{85} \mathrm{Rb} \text {. } \\
& \text { This experiment is performed in an atomic beam of }{ }^{85} 5_{\mathrm{Rb}} \text { and }{ }^{87} \mathrm{Rb} \text { atoms in }
\end{aligned}
$$
their ground state. The atoms are subjected to two laser excitations in the presence of an electric field. A cW single-mode high resolution dye laser right-handpolarized is tuned and servo-locked on the transition $5{ }^{2} \mathrm{~S}_{1 / 2} \mathrm{~F}=3 \rightarrow 5 \quad{ }^{2} \mathrm{P}_{3} / 2 \quad \mathrm{~F}=4$ of the isotope ${ }^{85} \mathrm{Rb}$. Consequently an optical-pumping between magnetic sublevels occurs and the use of a cw laser permits the excitation of ${ }^{85} \mathrm{Rb}$ atoms in the pure state $5^{2} \mathrm{P}_{3 / 2} \mathrm{~F}=\mathrm{H}_{\mathrm{F}}=4$, which corresponds to the well-defined magnetic orbital quantum number $m_{\ell=1}$. The ionizing light is obtained from a tunable pulsed dye laser which is $\sigma^{+}, \pi$ or $\sigma^{-}$polarized. The produced ions are counted by an electron multiplier. Spectra corresponding to the three different polarizations of the pulsed laser have been recorded (Fig. 9). In the energy range $\mathrm{E} \sim-200 \mathrm{~cm}^{-1}$ the structures of each of the three spectra can be classified in two different groups, the widths and the pxofiles being very similar in a given group. For example in the $\boldsymbol{\sigma}^{+} \sigma^{+}$spectrum one observes dissymmetrical structures looking like Fano profjles [16] with a parameter $q$ approximately equal to 1 ; between two such resonances there appears a very narrow symmetrical structure. Some resonances are located at the same energy in the three spectra but they correspond to very different profiles; for example the narrow structures of the $\sigma^{+} \sigma^{-}$spectrum are associated with the broad ones of the $\sigma^{+} \pi$ spectrum and reciprocally. By using the hydrogenic model we try to label the observed resonances by the parabolic quantum numbers ( $n$, $\left.n_{1}, m_{\ell}\right)$. If one assumes that $m_{\ell}$ is an exact quantum number, the $m_{\ell}$ values of the final state are respectively $0,+1$, and +2 for the $\sigma^{+} \sigma^{-}$, $\sigma^{+} \pi$ and $\sigma^{+} \sigma^{+}$ spectra. With this assumption, only one resonance out of two can be identified in each spectra. More precisely in the three spectra only the broad structures can be labelled. However recalling that there is an apparent degeneracy in the three spectra and that rubidium is a relatively heavy atom, it is possible to assert that the symmetry breaking with respect to $\mathrm{m}_{\ell}$ arises from the spin-orbit interaction which couples excited states with the same ${ }^{l} m_{j}$ value but with different $m_{\ell}$ values. The structures of the $\sigma^{+} \sigma^{-}, \sigma^{+} \pi$ and $\sigma^{+} \sigma^{+}$spectra are associated respectively with final states $m_{j}=1 / 2,3 / 2$ and $5 / 2$ and correspond to the two series $\left(m_{\mathfrak{s}}=1 / 2\right.$, $\left.m_{\ell}=m_{j}-1 / 2\right)$ and $\left(m_{s}=-1 / 2, m_{\ell}=m_{j}+1 / 2\right)$. Then all resonances observed in the three. spectra can be identified, and the two series correspond to different $m_{\mathfrak{s}}$ values: the broad structures are associated with $m_{S}=1 / 2$ meanwile the narrow resonances coxrespond to $m_{s}=-1 / 2$, independently of the studied spectrum. However in this analysis, the same identification $\left(n_{1} m_{\ell}\right)$ is ascribed to two resonances 


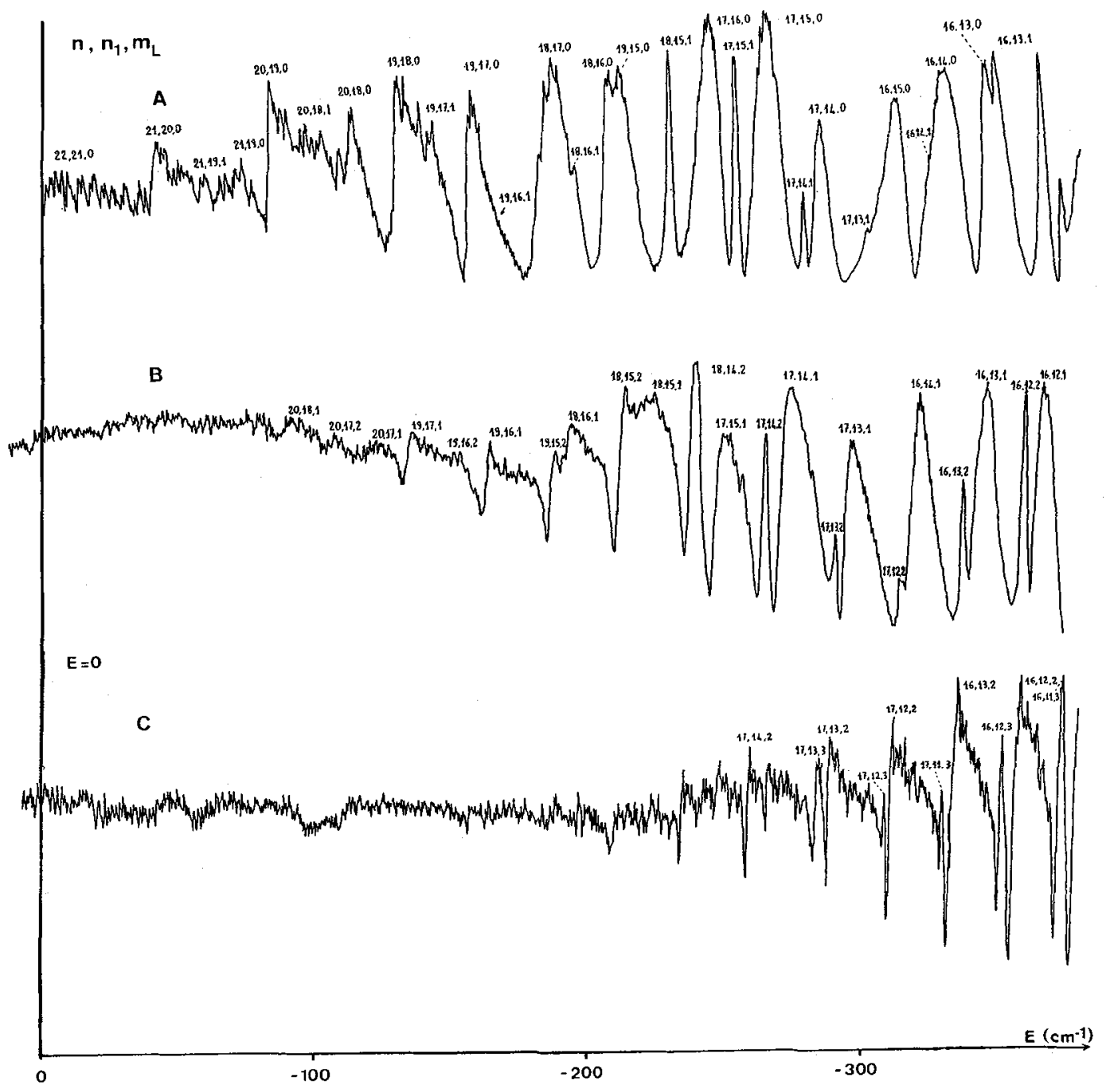

\section{Figure 9}

Recordings of the two-step photoionization spectra in the rubidium atom.

$$
\Phi=9950 \mathrm{~V} / \mathrm{cm} \text {. }
$$

Various light polarizations are used : A : $\sigma^{+} \sigma^{-}, \mathrm{B}: \sigma^{+} \pi, \mathrm{C}: \sigma^{+} \sigma^{+}$ $\left(\mathrm{n}, \mathrm{n}_{1}, \mathrm{~m}\right)$ : parabolic quantum numbers labelling the resonances. 
with very different profiles according to whether these structures are observed in the spectrum $m_{j}=m_{\ell^{+1 / 2}}$ or $m_{\ell^{-1 / 2}}$. As it was discussed previously, in a photoabsorption spectrum one does not observe the density of excited states but only the density of oscillator strengths, which depends strongly on the coupling between dis. crete and continuum states. In hydrogenic approximation, excited states associated with a given $m_{j}$ value consist both of continua $\left(\mathrm{E}, \mathrm{n}_{1}, \mathrm{~m}_{\ell}, \mathrm{m}_{\mathrm{s}}\right.$ ) associated with the wavefunctions $\Psi^{+}(E)$ and $\Psi^{-}(E)$ respectively for $m_{S}=1 / 2$ and $-1 / 2$, and of discrete states $\left(\mathrm{n}, \mathrm{n}_{1}, \mathrm{~m}_{\ell}, \mathrm{m}_{\mathrm{s}}\right)$ noted by $\varphi^{+}$and $\varphi^{-}$in the same way. In the rubidium atom these states interact through $\Delta V$, the non-coulombic part of the electrostatic potential, and through $\Lambda$ the spin-orbit interaction (Fig. 10), and the eigenfunction $\Phi(E)$ can be expanded in terms of $\varphi^{+}, \varphi^{-}, \Psi^{+}\left(E^{\prime}\right)$ and $\Psi^{-}\left(E^{\prime}\right)$. In the study of electric dipole transitions connecting the lower state ${ }^{2} \mathrm{P}_{3 / 2}$, $m_{S}^{\prime}=1 / 2, m_{\ell}^{\prime}=1$ to the upper state $\Phi(E)$, only the components $\Psi^{+}\left(E^{\prime}\right)$ and $\varphi^{f}$ of the wavefunction contribute to the value of the transition matrix element. So the width of the profile associated with the discrete state $\varphi^{+}$(or $\varphi^{-}$) is determined by the strength of the interaction $\Delta V+\Lambda$ (or $\Lambda$ ) connecting the studied state to the continuum $\Psi^{+}\left(E^{\prime}\right)$. The parameter $q[16]$ depends on the same interaction and on $d$ and $D$ the matrix elements of the transition operator connecting the lower state to $\varphi^{+}$and $\Psi^{+}\left(E^{\prime}\right)$ respectively. For the profile associated with $\varphi^{-}, q$ vanishes. We have studied a simple model consisting in three discrete

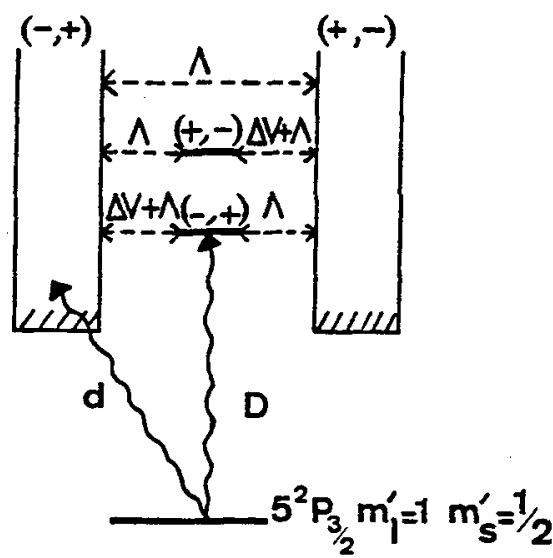

$$
\begin{array}{lll}
(-,+) & m_{1}=m_{j}-1 / 2 & m_{s}=1 / 2 \\
(+,-) & m_{1}=m_{j}+1 / 2 & m_{s}=-1 / 2
\end{array}
$$

Figure 10

Schematic representation of the discrete and continuous excited states with a given $\mathbf{m}_{j}$ value. The couplings are

$\Lambda$ the spin-orbit interaction.

$\Delta V$ the non-coulombic part of the central potential. states of energies $E_{1}, E_{2}$ and $E_{3}$ coupled to one continum. The states 1 and 3
correspond to states $\varphi^{+}$and are associated with the same parameters $\Gamma$ and $q$; the state 2 corresponds to state $\varphi^{-}\left(q_{2}=0\right)$. The resulting absorption profiles [16] are calculated and some results are reported on Fig. 11. To interpret qualitatively the $\sigma^{+} \sigma$ spectrum, we suppose that $\Gamma_{2}$ (due to the spin-orbit interaction of $p$ states) is smaller than $\Gamma_{1}$ (due to the non-hydrogenic character of $s$ states). For a relatively small value for $q_{1}$, the profile consists in relatively symmetrical structures, a narrow one lying between two broad resonances. If one assumes that $\Gamma_{1}=\Gamma_{2}$ and that $q_{1} \sim 1$, then the resulting profile reproduces the structures observed in the $\sigma^{+} \sigma^{+}$spectrum. Indeed for states $m_{j}=5 / 2$, the interactions $\Lambda$ and $\Delta V$ are small and of the same order of magnitude. This simple analysis clearly demonstrates that the Stark photoionization spectra of Rb are strikingly perturbed by the spin-orbit interaction, even in the vicinity of the zerofield ionization limit. Compared to the Stark photoionization spectra from the $3^{2} \mathrm{P}_{3 / 2} \mathrm{~m}_{l}=1$ state of Na [10] the structures are twice as numerous in the Rb 
a)

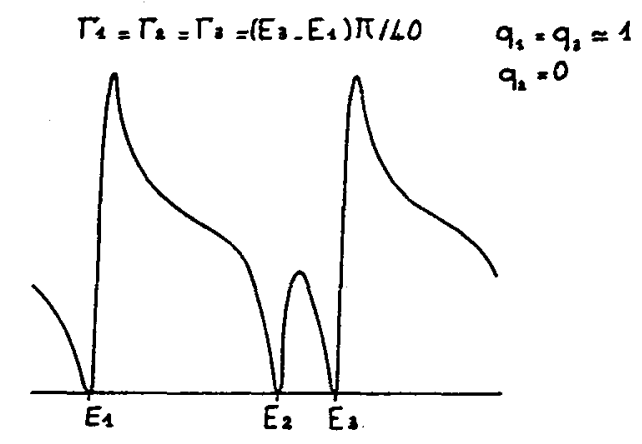

Figure 11

Profiles calculated in

the three-state model

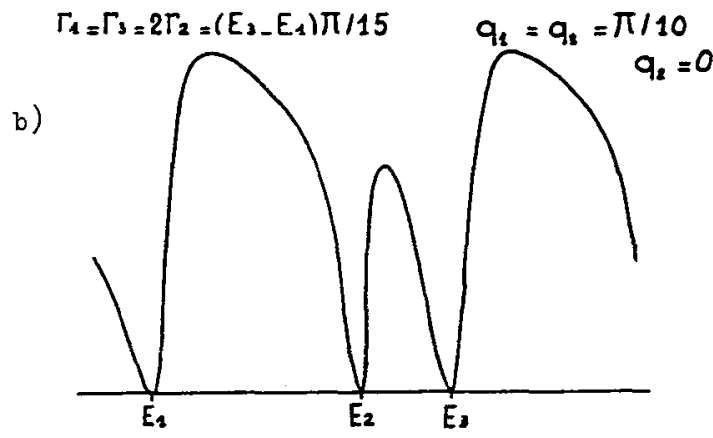

a) $\sigma^{+} \sigma^{+}$spectrum

b) $\sigma^{+} \sigma^{-}$spectrum

spectra, a result which can only be understood by taking into account the spin-orbit interaction.

However the perturbations $\Delta V$ and $\Lambda$ are so large that it is not possible to analyze the different structures independently from one another, by studying the coupling of a single discrete state with one continuum. It is necessary to consider as a whole the effects of the core interaction and those of the spin-orbit interaction. A suitable method has been developed within the frame-work of the non relatim. vistic multichannel quantum defect theory [18] and successfully applied to the analysis of the Stark photoionization spectrum of $\mathrm{Na} 3^{2} \mathrm{P}_{3 / 2}$ [19]. In this work only the effects due to the spherically symmetric ionic core are introduced through quantum defects. This coupling merely dilutes the resonances located near the zerofield ionization threshold without shifting their positions or creating new peaks.

In conclusion the experimental study of the Stark photoionization spectra of $\mathrm{Rb}$ has clearly shown the important role played by the spin-orbit interaction even near the zero-field ionization limit. Indeed, although weak, this coupling leads to the observation of new resonances in the photoionization of a atate with a welldefined $m_{\ell}$ value. The observed profiles depend strongly on the polarization of the light because the crucial quantity is not simply the density of excited states but the density of oscillator strengths. Furthermore at the anticrossing of states with different $m_{\ell}$ values, the spin-orbit interaction strongly perturbs the fieldionization properties of the states. This property can be explained by introducing explicitly both the energy and field dependences of the density of continuum states. Lastly non-hydrogenic interactions strongly modify the photoionization stark spectra : indeed, in the over critical region, the Stark spectrum of hydrogen consists simultaneousiy in discrete and continuum states, and all arguments deduced from the study of unperturbed bound states must be generalized with great caution. 


\section{References.}

[1] Helfrich, K., Theor. Chem. Acta 24 (1972) 271.

[2] Zimmerman, M. L., Littman, M. G., Kash, M. M. and Kleppner, D., Phys. Rev. A 20 (1979) 2251.

[3] Bethe, H. A. and Salpeter, E. E. (1957), Quantum Mechanics of One- and TwoElectron Atoms (New York Academic).

[4] Hatton, G. J., Phys, Rev. A 16 (1977) 1347.

[5] Luc-Koenig, E. and Bachelier, A., J. Phys. B 13 (1980) 1743 and 1768.

[6] Harmin, D. A., Phys. Rev. A 24 (1981) 2491.

[7] Feneuille, S., private communication.

[8] Freeman, R. R., Economou, N. P., Bjorklund, G. C. and Iu, K. T., Phys. Rev. Lett. 41 (1978) 1463.

Freeman, R. R. and Economou, N. P., Phys. Rev. A 20 (1979) 2356.

[9] Ink, T. S., Di Mauro, L., Bergeman, T. and Metcalf, H., Phys. Rev. Lett. 47 (1981) 83.

[10] Feneuille, S., Iiberman, S., Luc-Koenig, E., Pinard, J. and Taleb, A., Phys. Rev. A 25 (1982) 2853.

[11] Luc-Koenig, E. and Bachelier, A., Phys. Rev. Lett. 43 (1979) 921.

[12] Harmin, D. A. (1981) private communication.

[13] Littman, M. G., Kash, M. M. and Kleppner, D., Phys. Rev. Lett. 41 (1978) 103.

[14] Feneuille, S., Ijberman, S., Iuc-Koenig, E., Pinard, J. and Taleb, A., J. Phys. B 15 (1982) 1205.

[15] Barley, D. S., Hiskes, J. R, and Rivière, A. C., Nucl. Fus. 5 (1965) 41.

[16] Fani, U., Phys. Rev. 124 (1961) 1866.

[17] Iittman, M. G., Zimmerman, M. L. and Kleppner, D., Phys. Rev. Iett. 37 (1976) 486.

[18] Fano, U., Phys. Rev. A 24 (1981) 619.

[19] Harmin, D. A., Phys. Rev. Iett. 49 (1982) 128, and to appear in Phys. Rev. A (1982). 